\title{
Burden and Associated Factors of Coronavirus Disease (COVID-19) in Al-Buraimi Governorate, Oman
}

\author{
Hanan Al-Marbouai ${ }^{1,2}$, Muhammad Muqeet Ullah ${ }^{1}$, Amal Al-Nafisi ${ }^{2}$, Mostafa Elsayed Elnifily ${ }^{1}$, \\ Ahmed Yar Al-Buloshi ${ }^{1}$, Sami Saeed Almudaraa ${ }^{2} \&$ Eman Elsayed Abd-Ellatif ${ }^{3}$ \\ ${ }^{1}$ Directorate of Disease Surveillance and Control, Directorate General of Health Services, Al-Buraimi \\ Governorate, Ministry of Health, Oman \\ ${ }^{2}$ Field Epidemiology Training Program, Ministry of Health, Saudi Arabia \\ ${ }^{3}$ Department of Public Health \& community medicine, Faculty of Medicine, Mansoura University, Egypt \\ Correspondence: Hanan Al-Marbouai, Field Epidemiology Training Program, Ministry of Health, Saudi Arabia, \\ Directorate of Disease Surveillance and Control, Directorate General of Health Services, Al-Buraimi Governorate, \\ Ministry of Health, Oman. Tel: 96-896-554-959.
}

Received: July 16, 2021 Accepted: August 27, 2021 Online Published: September 8, 2021

doi:10.5539/gjhs.v13n10p61

URL: https://doi.org/10.5539/gjhs.v13n10p61

\begin{abstract}
The Coronavirus disease 2019, is a global pandemic that has brought a significant health challenge all over the world. Oman reported its first case of COVID-19 on 24 February 2020. Understanding patient characteristics and demand on the healthcare system is essential to ensuring Oman can continue to provide high quality care. The aim of this study is to describe the burden of COVID-19 and associated factors for more severe disease in Al-Buraimi Governorate, Oman. We retrieved demographic and clinical data from electronic medical records for all COVID-19 laboratory-confirmed patients in Al-Buraimi Governorate from February 1 to August 31, 2020. We assessed the factors for hospitalization and outcome (recovery/death) using descriptive statistics, chi-square test/fisher exact test, spearman's correlation, and multivariable logistic regression model in Epi info 7, Microsoft excel and SPSS software ( $\mathrm{p} \leq 0.05$ significance level). A total of 977 COVID-19 patients were identified, with a prevalence rate of 8.4 per 1000 in Al-Buraimi Governorate. The male: female ratio was 3.1:1. Of COVID-19 patients, $11.7 \%$ were hospitalized, and $1.5 \%$ died. Diabetes $(12.2 \%)$ and hypertension $(10.8 \%)$ were the most prevalent chronic conditions among COVID-19 patients. Older patients ( $>60$ years old) and those with comorbidities (chronic kidney disease, diabetes, heart disease, hypertension) were prone to hospitalization ( $\mathrm{p}$ $<0.001)$, intensive care $(\mathrm{p}<0.001)$, and death $(\mathrm{p}<0.001)$. Multivariate logistic regression analysis found that these risk factors were significantly associated with hospital admission $(\mathrm{OR}=5.905,95 \%$ CI 3.923-8.889; $\mathrm{p}<0.001)$, ICU admission $(\mathrm{OR}=4.363,95 \%$ CI $1.952-9.750 ; \mathrm{p}<0.001)$, and death $(\mathrm{OR}=6.785,95 \%$ CI $2.295-20.062$; $\mathrm{p}<0.001)$. A higher incidence of cases were observed among men and Omanis. Public health messaging for COVID-19 prevention should be tailored to inform these groups to slow the spread. Our findings are consistent with other studies, and local healthcare providers should be informed of the risk for severe disease among older patients and those with comorbidities, importance of early diagnosis, and prompt treatment.
\end{abstract}

Keywords: burden, Covid-19, mortality, risk factors, SARS-CoV-2

\section{Introduction}

The Coronavirus diseases 2019 (COVID-19) is triggered by the novel severe acute respiratory syndrome coronavirus 2 (SARS-CoV-2). The disease was first reported in December 2019 in Wuhan province and has since been declared by the world health organization as a major pandemic (Alandijany et al., 2020). Also, though the SARS-CoV-2 is in the same family as MERS-CoV and SARS-CoV, it has shown to be significantly different in how it quickly spreads, yet with a low mortality rate (about 3.4\%) compared to $10 \%$ for SARS-CoV and $34 \%$ for MERS-CoV. Similarly, the MERS-CoV and SARS-CoV have shown to have a zoonotic origin and are life-threatening diseases (Boukhatem, 2020). However, the virus being highly potent in transmission between humans, it has caused more deaths than the other strains. By June 8, 2021, the world had recorded over 174 million cases and over 3,700,000 deaths. In Oman, the situation has not been any better because more than 226,600 cases have been reported, with 2,434 deaths due to the virus (World Meters, 2020). The high number of cases has affected the patients and put the healthcare systems under extreme pressure. Besides, hospitals have been 
overwhelmed by patients resulting in deaths that could have been prevented in ordinary circumstances (World Health Organization, 2020).

On the other hand, evidence from studies done in countries like China, Europe, and the United States suggests a high risk of the COVID-19 being severe in individuals with underlying health issues. And according to the World Health Organization's definition of severe diseases, the patient often suffers from severe acute respiratory illness (this includes fever and at least one symptom of respiratory illness such as coughing or shortness of breath, and it requires hospitalization). Based on a study done in the United States, the underlying health conditions were reported in about $70 \%$ of the people hospitalized due to COVID-19 (Clark et al., 2020). Similarly, about $94 \%$ of deaths in the US have been as a result of an underlying health issue. In light of this, WHO has issued a list of people at a higher risk of having severe COVID-19. These include cardiovascular disease, chronic kidney disease, diabetes, chronic respiratory disease, among other chronic conditions. When a person has such illnesses, there is an increased risk of hospitalization and a ventilator for oxygen supplementation. Also, a significant proportion of the additional healthcare burden of Coronavirus is likely to occur in people with underlying conditions (Clark et al., 2020).

Consequently, an increasing body of evidence suggests that persons aged 65 and above are at a greater risk of severe Coronavirus (Ahrenfeldt et al., 2020). For example, a study in China found that the hazardous ratio of reaching the composite endpoint (such as being admitted into the intensive care unit, using a ventilator, or even mortality) was high for middle-aged and elderly patients with comorbidities (Ahrenfeldt et al., 2020). Besides, other studies have suggested that COVID-19 has more severe symptoms and mortality in men than women (Park, 2020). Despite this, it is still unknown whether there is a difference in the immune system response against SARS-CoV-2 in these two sexes (Takahashi et al., 2020). It would also be pivotal to establish if such differences exist; they would correlate with the sex difference in the disease course of Coronavirus. However, researchers have shown that male patients have higher plasma levels of innate immune cytokines, while female patients exhibited more aggressive $\mathrm{T}$ cell activation than male patients (Takahashi et al., 2020). Such findings are revolutionary and might help explain the sex differences in the severity of COVID-19. Thus, future studies must establish if such sex biases only exist in certain geographical regions or whether they can be identified in other regions such as Oman.

\subsection{Rationale}

From the literature review conducted, it is evident that a lot needs to be done to better understand the epidemiological and etiological factors of COVID-19. In particular, the burden and risk factors of COVID-19 vary significantly from one region to the other, even in people of the same age group, sex, race, among other demographics (Rod et al., 2020). Therefore, there is a need to conduct a study on the burden and the risk factors of COVID-19 in Oman as most of the existing studies on the same area are conducted in other regions of the world, thus limiting the external validity of the burden and risk factors for other countries.

This study will be among the first few studies to investigate the burden of COVID-19 in Oman and the Middle East; therefore, it will add knowledge to the existing body of research on the burden and risk factors of COVID-19. Also, critical information will be availed to healthcare providers and policymakers on the various mechanisms and policies needed to protect the population at risk of developing other illnesses after getting the virus. Th rough these findings of this study; the government will estimate the number of people at increased risk of COVID-19 infections and the care needed to prevent or mitigate these vulnerabilities. Most importantly, the government will be able to design a more effective intervention program that will protect vulnerable people and ease the pressure on healthcare systems. Lastly, the results will inform a larger assent of the health, social, and economic implications of shielding various groups (Rod et al., 2020).

\subsection{Overview of the Current Literature}

Coronavirus disease 2019 has proven to be a significant global crisis prompting a World Health Organization (WHO) declaration that this illness is now a pandemic (Azarpazhooh et al., 2020). The first two cases of COVID-19 were reported in Oman on February 24 from the Muscat governorate, the capital of Oman (Khamis et al., 2020). After discovering these cases, policymakers and healthcare experts have struggled to contain the massive spread of infection (Alandijany et al., 2020). In Oman, researchers sought to establish the epidemiological characteristics of the first 1304 cases of COVID-19. Their study included all the patients from laboratory-confirmed cases between February 24 and April 17, 2020. Their findings conformed to similar data obtained in Italy and the United States, where it has been found that men have had higher rates of confirmed cases compared to females. Nonetheless, the infection pattern was in contrast with studies done in China, where the number of infected females was higher than men. It was also noted that the fatality rate in Oman was higher in men compared to women. Although the explanations for these variations are unknown, it has been suggested that men 
are more exposed to work and have higher comorbid conditions that may worsen their prognosis (Khamis et al., 2020).

In the Gulf Region, the Gulf Cooperation Council (GCC) countries have done a commendable job in flattening the infection curve and stopping the symptom progression of COVID-19 in the vulnerable population. This has been achieved through the implementation of proactive plans and timely decisions in responding to the outbreak. When the first case of COVID-19 got announced in GCC countries, the countries responded by initiating strict control measures to prevent the spread of the infection. For example, most countries in the gulf region treated all the cases of COVID-19, whether declared or suspected, as a health emergency. Further, free healthcare to all COVID-19 patients was provided, followed by immediate closure of schools and universities, and raising awareness campaigns and suspension of all flights. These aggressive measures may not have halted the spread entirely but massively succeeded in bringing the spread under control. Also, awareness complaints about COVID-19 were made through the media, and public cooperation helped significantly to contain the infections (Alandijany et al., 2020).

The burden of disease due to COVID-19 has been an area that has interested scientists since the outbreak became a global pandemic. A study on the burden of illness due to COVID-19 in Korea using disability-adjusted life years found that the years of life lived with disability (YLDs) were significantly higher in females than in males, but years of life lost (YLLs) were higher in male compared to the female. Also, the total disease burden associated with COVID-19 in South Korea at the time of this research was about 2,531.0 DALYs and 4.930 DALYs per 100,000 population. This study also revealed that the DALYs per 100,000 people were highest in the age group more than 80 years, closely followed by 70-79, 60-69, and 50-59 years (Jo et al., 2020).

In ongoing research, preliminary data show that COVID-19 and cardiovascular disease share some pathomechanism risk factors such as ACE2 receptor invasion and renin-angiotensin system signaling, oxidative stress, systemic inflammation, and endothelial dysfunction. Besides, the study has already shown that COVID-19 is likely to cause dyslipidemia, dysglycemia, kidney and liver disease. These mechanisms and illnesses cause the risk factors of various conditions such as diabetes mellitus, hypertension, obesity, atherosclerosis, and respiratory tract infections. In the real sense, it is becoming increasingly evident that the prevalence of common risk factors/comorbidities between coronavirus and cardiovascular diseases occurrence of ACE2 receptors on the endothelium, and thus bringing about the pathomechanism of COVID-19 infection suggests that the Coronavirus may increase the burden of caring for patients with chronic illnesses (Ogeng'o et al., 2020).

With the SARS-CoV-2019 straining healthcare facilities worldwide to a critical point, there has been a clear need to strengthen the primary healthcare systems as an effective policy of protecting people towards health emergencies, achieving universal health care, and promoting wellbeing. In particular, the SARS-CoV-2019 has served as a cruel reminder that non-communicable diseases (NCDs) are closely interlinked to the burden of communicable diseases, which increase morbidity and mortality (Kraef et al., 2020). In Oman, NCDs account for about $68 \%$ of the total deaths, and $18 \%$ of these deaths occur to people between 30 to 70 years (World Health Organization, 2017). Based on a report that the United Nations Interagency Task Force availed on the Prevention and Control of Non-communicable Diseases (NCDs) in 2016, it was found that the main risk facing most Omani adults is the insufficient intake of fruits and vegetables. More than $40 \%$ of Omani adults are physically inactive, while 1 in 7 means using tobacco. About $40 \%$ of Omani adults have hypertension, and $12 \%$ have been diagnosed with diabetes (World Health Organization, 2017).

\subsection{Objectives}

1) To estimate the prevalence of COVID-19 cases in 3 Wilayats of Al Buraimi Governorate, Oman, from February 1, 2020, to August 31, 2020.

2) To identify the common factors associated with COVID-19 infection among positive cases from February 1, 2020, to August 31, 2020.

3) To identify and measure the common factors associated with admission and death of COVID-19 cases from February 1, 2020, to August 31, 2020.

\section{Methodology}

\subsection{Study Setting}

The study was conducted in Al-Buraimi Governorate, Oman, from February 1, 2020, to August 31, 2020. The governorate consists of three wilayats (Provinces); Al Buraimi, Mahdah, and Al-Sunaynah. The total population of the Al-Buraimi Governorate is 115,658 persons. 


\subsection{Study Design}

The study was an analytical cross-sectional study to determine the prevalence of COVID-19 and the secondary purpose of establishing the associated factors of all positive cases reported in the Al-Buraimi Governorate.

\subsection{Participants}

2.3.1. Inclusion criteria: The study included all the confirmed positive cases of COVID-19, who were residents of Al-Buraimi Governorate, from February 1, 2020, to August 31, 2020.

2.3.2 Exclusion criteria: All positive cases of COVID-19 who belong to other governorates were excluded from the study.

\subsection{Sampling Technique}

The study data was record-based, including the whole sample of all positive cases of COVID-19 in Al-Buraimi Governorate, from February 1, 2020, to August 31, 2020.

\subsection{Data Collection}

The data was record-based obtained from Omani Ministry of Health's approved applications (Ministry of Health e-notification system and Electronic Al-Shifa system), that are comprehensive and flexible online-based platforms aiming at accommodating Omani programs for public health to facilitate data collection. Also, they allow public health professional to collect data, predict the distribution of the diseases, and prevent and control the distribution of the diseases and provide decision-makers with evidence-based results extraction tool.

\subsection{Variables}

The variables included in the study were age, gender, nationality, risk factors, type of quarantine, admission, source of infection, outcome.

- Independent variables: age, gender, nationality, type of quarantine, source of infection

- Dependent variables: risk factors, outcome, admission

\subsection{Study Duration}

The total study took about ten months, starting from September 2020. Data collection and analysis took about six months, while writing report took around 4 months.

\subsection{Data Analysis}

Epi Info seven, SPSS, and Microsoft Excel were used for data entry and analysis. To assess the prevalence of COVID-19, we analyzed and cross-reference the available data as obtained from Al-Shifa, and MOH-approved applications (Tarassud) for validation and reliability. Descriptive statistics were performed by calculating percentage, frequency, means, standard deviation, and rate of infection. The associated factors were assessed using the Chi-square test in Epi info 7 with a p-value $\leq 0.05$ significance level. The regression test we used was logistic regression which was the appropriate regression analysis to conduct in this case. Like all regression analyses, logistic regression is a predictive analysis.

\section{Ethical Declaration}

The ethical approval was obtained from the Oman National Bioethics Committee (NBC). The research used data of patients who have tested positive for COVID-19, patient privacy was the main ethical concern. However, the study ensured that no personal information was revealed, and that the data obtained was used only for study purposes.

\section{Results}

\subsection{Demographic Characteristics}

Data of 977 covid-19 patients of Al-Buraimi Governorate was obtained for retrospective analysis. Table 1 represents the demographic profile of COVID-19 cases reported during the pandemic in Al-Buraimi, Oman, from Feb to Aug 2020. The mean age of the sample size was 35.7 years, with a standard deviation of 13.4. Most of the reported cases fall into the age category of 30-59 (60.5\%) followed by the age categories of 15-29 years (29.4\%), 60 years or above $(6.1 \%), 5-14$ years $(2.7 \%)$, and $0-4$ years $(1.3 \%)$, respectively. The majority of the patients were male at $75.5 \%$, and about $56.3 \%$ of the sample size being Omani nationals, while the remaining percentage was composed of expatriates. Further, the prevalence of COVID-19 patients was 95.2\% in Al Buraimi Wilayat, 3.7\% in Mahdha Wilayat and $1.1 \%$ in Al Sunaina (Table 1). 
Table 1. Summary of COVID-19 Cases According to Demographic Characteristics, Al-Buraimi, Oman, and February to August 2020

\begin{tabular}{|c|c|c|c|}
\hline \multirow{2}{*}{ Variables } & & \multicolumn{2}{|c|}{ Total } \\
\hline & & $\mathbf{N}$ & $\%$ \\
\hline \multirow{7}{*}{ Age Category } & $0-4$ Years & 13 & $1.3 \%$ \\
\hline & 5-14 Years & 26 & $2.7 \%$ \\
\hline & 15-29 Years & 287 & $29.4 \%$ \\
\hline & 30-59 Years & 591 & $60.5 \%$ \\
\hline & 60 Years and Above & 60 & $6.1 \%$ \\
\hline & Mean & \multicolumn{2}{|c|}{35.7} \\
\hline & Std Dev & \multicolumn{2}{|c|}{13.4} \\
\hline \multirow{2}{*}{ Sex } & Male & 738 & $75.5 \%$ \\
\hline & Female & 239 & $24.5 \%$ \\
\hline \multirow{2}{*}{ Nationality } & Omani & 550 & $56.3 \%$ \\
\hline & Non-Omani & 427 & $43.7 \%$ \\
\hline \multirow{3}{*}{ Wilayat } & Al Buraimi & 930 & $95.2 \%$ \\
\hline & Mahdha & 36 & $3.7 \%$ \\
\hline & Al Sunaina & 11 & $1.1 \%$ \\
\hline
\end{tabular}

From the three Wilayats of Al-Buraimi Governorate, a total population of 115,658 was available. From this, about 977 persons had been positively confirmed to have Covid-19. This was an 8.4 cases prevalence rate in every 1000 persons. Breaking these cases according to a specific Wilayat, it was found that Al Buraimi Wilayat, which has 107, 411 people had 930 cases, and this constituted about 8.7 cases per 1000 people. Mahdha had 36 cases from a population of 7,408 persons with prevalence rate of 4.9 per 1000 people, while Al Sunaina had 11 cases from 839 people, making its prevalence rate at 13.1 per 1000 cases. From these statistics, it is evident that Al Buraimi had most cases, and Al Sunaina had the highest prevalence, that might be due to the lower population of Al-Sunaina Wilayat (Table 2).

Table 2. Prevalence of COVID-19 Cases According to Wilayat, Al-Buraimi, Oman, February to August 2020

\begin{tabular}{lllc}
\hline Variables & Total Population & Number of Cases & Prevalence \\
\hline Al-Buraimi Governorate & 115658 & 977 & 8.4 \\
Wilayat Al Buraimi & 107411 & 930 & 8.7 \\
Wilayat Mahdha & 7408 & 36 & 4.9 \\
Wilayat Al Sunaina & 839 & 11 & 13.1 \\
\hline
\end{tabular}

To understand the distribution of cases within the study time frame, we conducted an analysis of the reported cases based on each epidemiological week. The first case in Al-Buraimi was reported on Mar 27, 2020, in the $13^{\text {th }}$ epidemiological week. In the first few weeks, the rate of positive case identification was low, which started to see a gradual increase towards the $19^{\text {th }}$ week. Most cases were recorded from $26^{\text {th }}$ to $30^{\text {th }}$ weeks, the highest number of cases were reported in week 30 (137 cases) followed by week 26 (134 cases), and week 28 (102 cases). There was significant decline in weekly cases after week 30 , until the end of our study, which was in August ( $36^{\text {th }}$ week). Some factors that could help explain the sharp changes in cases per week include change in case definition and various containment measures instituted by the government, such as lockdowns and mandatory wearing of masks, among others. Towards the onset of the outbreak, there was little information available on how to slow the spread. But as research and field data became available, various containment measures were recommended, and their impact can be seen in the Figure 1. 


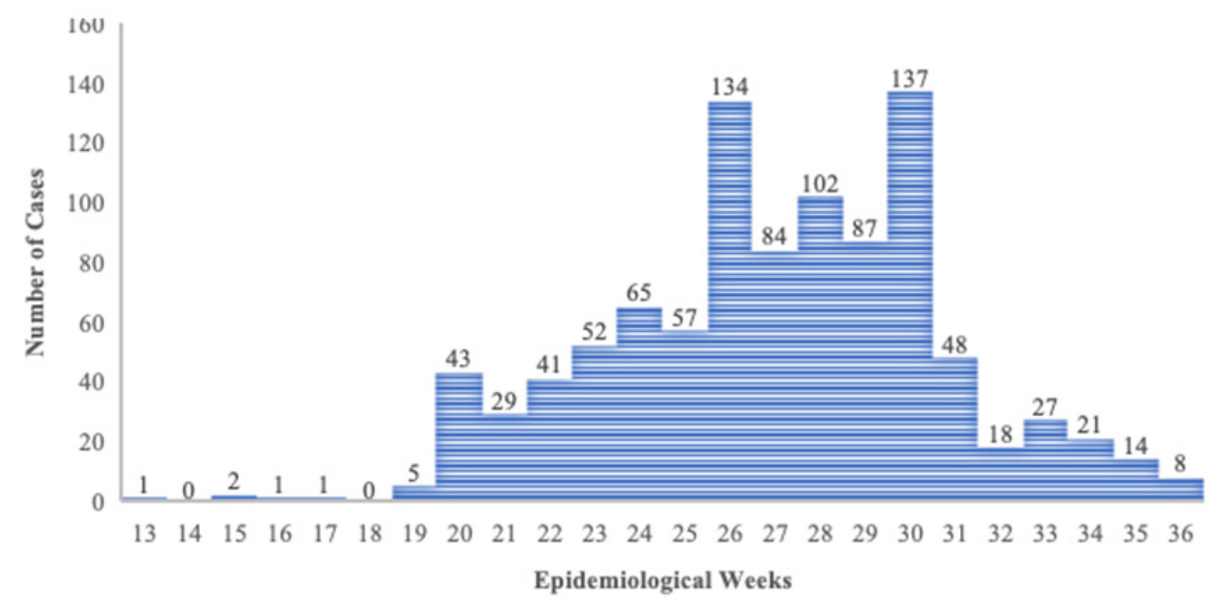

Figure 1. Distribution of COVID-19 Cases According to Epidemiological Week, Al-Buraimi, Oman, February to August 2020

\subsection{Risk Factors Related to Covid-19 Cases}

To understand the distribution of the risk factors in the Covid-19 cases, the underlying illnesses in the patient who tested positive were recorded. From this record, the highest number of patients, 119 patients (12.2\%), were found to have diabetes, and closely followed by hypertension at 106 patients $(10.8 \%)$. Age more than 60 years old was a third at $53(5.4 \%)$, followed by Asthma at $27(2.8 \%)$. The lowest cases indicated in risk factors were chronic kidney disease (CKD) and Hypothyroidism, both with a 1.0\% occurrence of COVID cases. OTHERS risk factors included chronic hematological disorder, chronic liver disease, chronic lung disease, immunocompromised, thyrotoxicosis, dyslipidemia, hepatitis $\mathrm{C}$, cancer, neurological disease, and rheumatological diseases. Combined, they accounted for $42(4.3 \%)$ of the cases. These percentages were calculated from the total of 977 patients to develop the overall understanding of the presence of COVID-19 cases with risk factors in total cases (Figure 2).

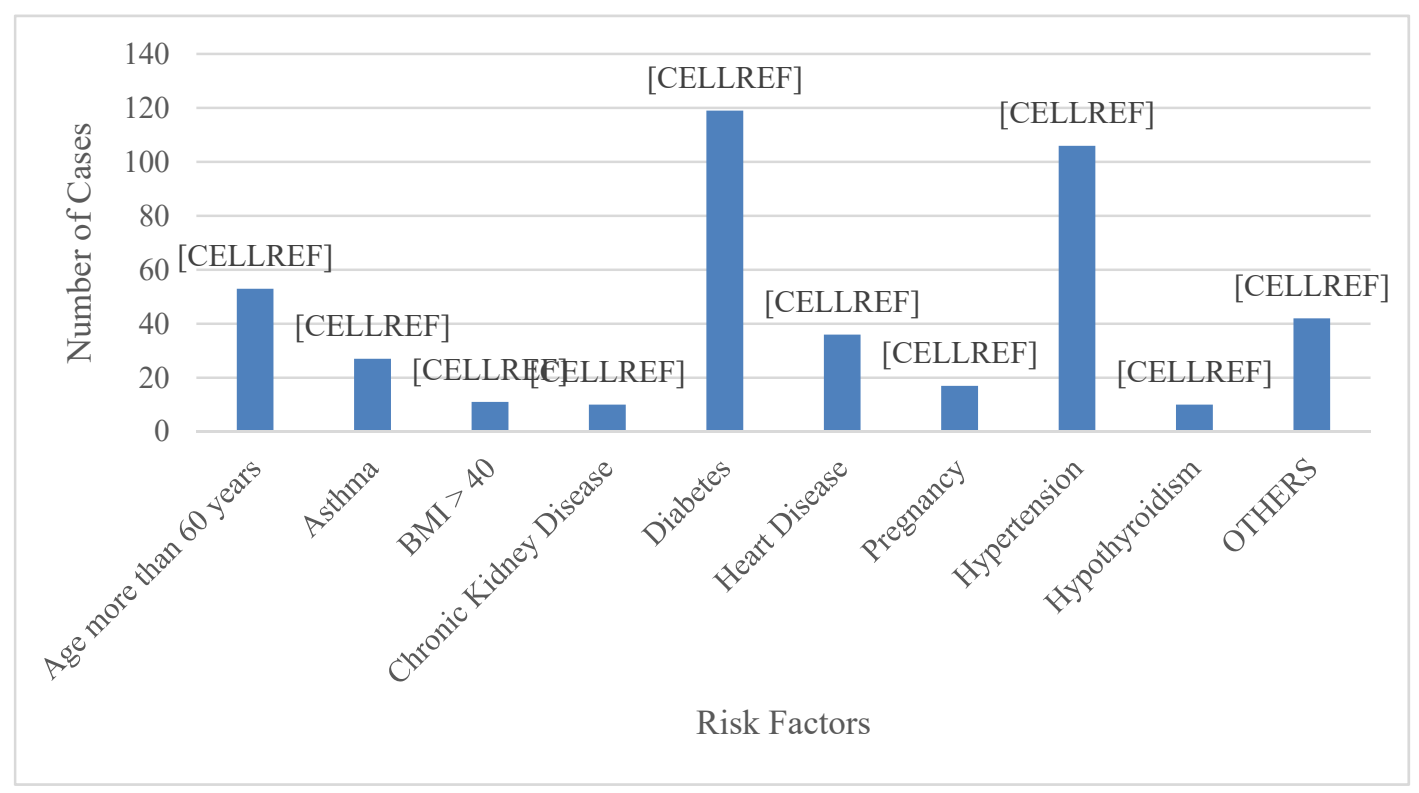

Figure 2. The Distribution of COVID-19 Cases According to Risk Factors, Al-Buraimi, Oman, and February to August 2020

*OTHERS include chronic hematological disorder, chronic liver disease, chronic lung disease, immunocompromised, thyrotoxicosis, dyslipidemia, hepatitis $\mathrm{C}$, cancer, neurological disease, and rheumatological diseases. 
From the sample size of the 977 patients, about 232 had one or more underlying conditions that could be attributed as a Covid-19 risk factor. However, about 745 had no risk factors. Out of the total sample, about $11.7 \%$ of patients had been admitted to the hospital, while about $88.3 \%$ were not admitted. Of the $11.7 \%$ of the admission (114 patients), 25 had proceeded to the intensive care unit. Besides the total covid-19 sample size, 962 patients had recovered, and 15 patients had died due to the virus or its related complications, hence, the death rate was $1.5 \%$ at the time (Table 3).

Table 3. Summary Table of COVID-19 Cases According to Risk Factor, Hospital Admission, ICU Admission and Outcome, Al-Buraimi, Oman, February to August 2020

\begin{tabular}{llcc}
\hline Variables & & Frequency & Percentage \\
\hline \multirow{2}{*}{ Risk Factor } & Yes & 232 & $23.8 \%$ \\
& No & 745 & $76.3 \%$ \\
\hline \multirow{2}{*}{ Hospital Admission } & Yes & 114 & $11.7 \%$ \\
& No & 863 & $88.3 \%$ \\
\hline \multirow{2}{*}{ ICU Admission } & Yes & 25 & $2.6 \%$ \\
& No & 952 & $97.4 \%$ \\
\hline \multirow{2}{*}{ Outcome } & Recovered & 962 & $98.5 \%$ \\
& Died & 15 & $1.5 \%$ \\
\hline
\end{tabular}

7.3 Correlation between Risk Factors Presentation vs. Hospital Admission, ICU Admission, and Outcome of COVID-19 Cases

To determine whether there is a collation between the risk factors and the likelihood of being admitted to a hospital, requiring the intensive care unit, or the outcome of the covid-19 cases, we performed a risk factor regression analysis. The p-value for the null hypothesis was set at 0.05 , meaning that any $p$-value equal to or above the null hypothesis's value would be proved to be true. The variable tested included hospital admission, ICU admission, and outcome, where all these variables had a p-value of less or equal to 0.0001 . Thus, there was a very strong correlation between all the three variables with the risk factors presentation (Table 4).

Table 4. Risk Factors Presentation vs. Hospital Admission, ICU admission, and Outcome of COVID-19 Cases, Al-Buraimi, Oman, February to August 2020

\begin{tabular}{|c|c|c|c|c|}
\hline \multirow{2}{*}{ Variables } & & \multicolumn{2}{|c|}{ Risk Factor } & \multirow{2}{*}{ P-Value } \\
\hline & & No & Yes & \\
\hline \multirow{2}{*}{ Hospital Admission } & No & 698 & 165 & \multirow{2}{*}{$<0.0001$} \\
\hline & Yes & 47 & 67 & \\
\hline \multirow{2}{*}{ ICU Admission } & No & 36 & 53 & \multirow{2}{*}{0.0001} \\
\hline & Yes & 11 & 14 & \\
\hline \multirow{2}{*}{ Outcome } & Died & 5 & 10 & \multirow{2}{*}{$<0.0001$} \\
\hline & Recovered & 740 & 222 & \\
\hline
\end{tabular}

A Chi-square test/fisher's exact test was used to determine the association between each risk factor and hospital admission. In these statistical analysis, we found that the highest risk factors associated with hospital admission were evident in people aged more than 60 years (chi square 54.73), chronic kidney disease (fisher exact test .0003), heart disease (chi square 69.85), diabetes (chi square 63.31), and hypertension (chi square 96.33), all at a p-value of less than 0.001, while Asthma showed significance with a p-value of 0.019 and BMI $>40$ showed significance with a p-value $<0.01$. Other illnesses such as pregnancy (p-value 0.99 ) and hypothyroidism (p-value 0.409) showed a strong to a near against null hypothesis correlation. However, illnesses are categorized as Others had a p-value of 0.012 , meaning they presented a significant threat to hospital admission (Table 5). In conclusion, it can be inferred that the results are significant, and it is safe to say that the presence of risk factors is associated with hospital admission of COVID-19 patients. 
Table 5. Association of Risk Factors Presentation vs. Hospital Admission of COVID-19 Cases by Using Chi-square, Fisher's Exact Test and P-Value, Al-Buraimi, Oman, February to August 2020

\begin{tabular}{lllccc}
\hline \multirow{2}{*}{ Risk factors } & \multicolumn{2}{l}{ Hospital Admission } & \multirow{2}{*}{ Chi-Square/ Fisher's Exact Test } & P-Value \\
\cline { 2 - 5 } & Yes & No & 54.73 & $<0.0001$ & $<0.0001$ \\
\hline Age more than 60 years & 23 & 30 & 5.48 & 0.03 & 0.019 \\
Asthma & 7 & 20 & 6.58 & 0.03 & 0.01 \\
BMI > 40 & 4 & 7 & 22.9 & 0.0003 & $<0.0001$ \\
Chronic Kidney Disease & 6 & 4 & 63.31 & $<0.0001$ & $<0.0001$ \\
Diabetes & 40 & 79 & 69.85 & $<0.0001$ & $<0.0001$ \\
Heart Disease & 20 & 16 & 0 & 1 & 0.99 \\
Pregnancy & 2 & 15 & 96.33 & $<0.0001$ & $<0.0001$ \\
Hypertension & 43 & 63 & 0.68 & 0.329 & 0.409 \\
Hypothyroidism & 2 & 8 & 6.28 & 0.23 & 0.012 \\
OTHERS* & 10 & 32 & & \\
\hline
\end{tabular}

Fisher exact test rather than chi square. Expected less than 5.

OTHERS* includes chronic hematological disorder, chronic liver disease, chronic lung disease, immunocompromised, thyrotoxicosis, dyslipidemia, hepatitis c, cancer, neurological disease, and rheumatological diseases.

When looking at the likelihood of ICU admission of patients with various risk factors, this study established that there was a significant association ( $p$-values $<0.0001$ ) of ICU admission for patients whose age was more than 60 years at a chi-square value of 35.32, had chronic kidney disease at a fisher exact value of .002, diabetes (chi square 13.61), heart disease (chi square 19.24) and hypertension at (chi square 16.78). Risk factors with the least threat of progression to ICU included Asthma at a fisher exact test value of 0.508 , BMI $>40$ (0.249), pregnancy (1), Hypothyroidism (0.229), and others (1) (Table 6). These risk factors can be considered as potential predictors of developing enough severe conditions to be admitted to ICU.

Table 6. Association of Risk Factors Presentation vs. ICU Admission of COVID-19 Cases by Using Chi-square, Fisher's Exact Test and P-Value, Al-Buraimi, Oman, February to August 2020

\begin{tabular}{lllccc}
\hline \multirow{2}{*}{ Risk factors } & \multicolumn{2}{l}{ ICU Admission } & \multirow{2}{*}{ Chi-Square/ Fisher's Exact Test } & P-Value \\
\cline { 2 - 5 } & Yes & No & 35.32 & $<0.0001$ & $<0.0001$ \\
\hline Age more than 60 years & 8 & 45 & 0.15 & 0.508 & 0.702 \\
Asthma & 1 & 26 & 1.9 & 0.249 & 0.168 \\
BMI > 40 & 1 & 10 & 30.51 & 0.002 & $<0.0001$ \\
Chronic Kidney Disease & 3 & 7 & 13.61 & 0.002 & $<0.0001$ \\
Diabetes & 9 & 110 & 19.24 & 0.002 & $<0.0001$ \\
Heart Disease & 5 & 31 & 0.45 & 1 & 0.5 \\
Pregnancy & 0 & 17 & 16.78 & 0.001 & $<0.0001$ \\
Hypertension & 9 & 97 & 2.24 & 0.229 & 0.134 \\
Hypothyroidism & 1 & 9 & 0.01 & 1 & 0.941 \\
OTHERS* & 1 & 41 & & \\
\hline
\end{tabular}

Fisher exact test rather than chi square. Expected less than 5.

OTHERS* includes chronic hematological disorder, chronic liver disease, chronic lung disease, immunocompromised, thyrotoxicosis, dyslipidemia, hepatitis c, cancer, neurological disease, and rheumatological diseases.

The impact of risk factors on the outcome is as important as their association with hospital admission and ICU admission. In this category, the outcome was based on the patients who died and those who recovered. Age more than 60 years had the strongest correlation to the outcome of death ( $\mathrm{P}$ value $<0.0001$ ), where it had a chi-square 
value of 68.15. It was closely followed by heart disease (chi square 56.61) and chronic kidney disease (fisher exact test $<0.0001$ ), both with $\mathrm{P}$ value $<0.0001$. Hypertension had a significantly higher correlation ( $\mathrm{P}$ value $<0.0001$ ), with a chi square of 13.38, while diabetes, hypothyroidism, and BMI $>40$ had moderate risk ( $\mathrm{P}$ value $<0.05)$, at fisher exact test value of $0.012,0.003$, and 0.157 , respectively. There were non-significant association of death in patients with Asthma, Pregnancy, and other conditions, with $p$ value $>0.05$ (Table 7). Though the percentage of recovered patients is high as compared to died patients it is still worth considering these predictors in patients with COVID-19.

Table 7. Association of Risk Factors Presentation vs. Outcome of COVID-19 Cases by Using Chi-square, Fisher's Exact Test and P-Value, Al-Buraimi, Oman, February to August 2020

\begin{tabular}{|c|c|c|c|c|c|}
\hline \multirow{2}{*}{ Risk factors } & \multicolumn{2}{|l|}{ Outcome } & \multirow{2}{*}{ Chi-Square/ } & \multirow{2}{*}{ Fisher's Exact Test } & \multirow{2}{*}{ P-Value } \\
\hline & Recovered & Died & & & \\
\hline Age more than 60 years & 45 & 8 & 68.15 & $<0.0001$ & $<0.0001$ \\
\hline Asthma & 27 & 0 & 0.43 & 1 & 0.511 \\
\hline $\mathrm{BMI}>40$ & 10 & 1 & 4.2 & 0.157 & 0.04 \\
\hline Chronic Kidney Disease & 7 & 3 & 54.15 & $<0.0001$ & $<0.0001$ \\
\hline Diabetes & 114 & 5 & 6.37 & 0.027 & 0.012 \\
\hline Heart Disease & 30 & 6 & 56.61 & $<0.0001$ & $<0.0001$ \\
\hline Pregnancy & 17 & 0 & 0.27 & 1 & 0.603 \\
\hline Hypertension & 100 & 6 & 13.38 & 0.003 & $<0.0001$ \\
\hline Hypothyroidism & 9 & 1 & 4.79 & 0.144 & 0.029 \\
\hline OTHERS* & 42 & 0 & 0.68 & 1 & 0.408 \\
\hline
\end{tabular}

Fisher exact test rather than chi square. Expected less than 5.

OTHERS* includes chronic hematological disorder, chronic liver disease, chronic lung disease, immunocompromised, thyrotoxicosis, dyslipidemia, hepatitis c, cancer, neurological disease, and rheumatological diseases.

Spearman's correlation was used to measure the correlation of risk factors with Hospital admission, ICU admission, and outcome of COVID-19. The spearman correlation showed that the probability for a person with the highlighted risk factors to be admitted at the hospital is at $.296^{* *}$, while progression to ICU admission was at $.125^{* *}$ and for death was $.127^{* *}$. These correlations of predictors with all three variables are quite low but the results are significant with a p-value $<0.001$ (Table 8 ). The low correlation might be explained by population size and the number of cases (having risk factors) admitted to hospital and ICU.

Table 8. Correlation between Risk Factors Presentation with Hospital Admission, ICU Admission and Outcome of COVID-19 Cases, Al-Buraimi, Oman, February to August 2020

\begin{tabular}{lcc}
\hline \multirow{2}{*}{ Variables } & \multicolumn{2}{c}{ Risk Factor } \\
\cline { 2 - 3 } & Spearman's Correlation & Sig. (2-tailed) \\
\hline Hospital Admission & $.296^{* *}$ & $<0.001$ \\
ICU Admission & $.125^{* *}$ & $<0.001$ \\
Outcome & $.127^{* *}$ & $<0.001$ \\
\hline
\end{tabular}

**. Correlation is significant at the 0.01 level (2-tailed).

To get a clearer understanding of risk factors' effect on hospital admission, ICU, and outcome, logistic regression analysis was conducted. B represents the coefficient that tells how many times the presence of 1 risk factor will affect a particular variable, for instance, the presence of 1 risk factor will increase the hospital admission, ICU admission and death by $1.776,1.473$, and 1.915 times, respectively. The regression results are significant with a p-value $<0.001$. $\operatorname{Exp}(\mathrm{B})$ shows the exponent of B which can be referred to as the odds ratio. The lower and upper confidence levels of $\operatorname{Exp}(\mathrm{B})$ with $95 \%$ confidence interval (C.I) can also be seen in Table 9 which gives the idea of the lower and upper $\mathrm{CI}$ for odds ratio. The odds ratio tells the odds of occurring of a particular event in one group 
compared to the opposite event in another group i.e., odds of hospital admission, ICU admission and death in COVID-19 patients with risk factors is $5.905,4.393,6.785$, respectively, as compared to COVID-19 patients without the presence of any risk factor.

Table 9. Regression between Risk Factors Presentation and Hospital Admission, ICU Admission, and Outcome of COVID-19 Cases, Al-Buraimi, Oman, February to August 2020

\begin{tabular}{|c|c|c|c|c|c|}
\hline \multirow{2}{*}{ Variables } & \multirow{2}{*}{ B } & \multirow{2}{*}{ Sig. } & \multirow{2}{*}{$\operatorname{Exp}(B)$} & \multicolumn{2}{|c|}{$95 \%$ C.I. for $\operatorname{EXP}(B)$} \\
\hline & & & & Lower & Upper \\
\hline Hospital Admission & 1.776 & $<0.001$ & 5.905 & 3.923 & 8.889 \\
\hline ICU Admission & 1.473 & $<0.001$ & 4.363 & 1.952 & 9.750 \\
\hline Death & 1.915 & $<0.001$ & 6.785 & 2.295 & 20.062 \\
\hline
\end{tabular}

\subsection{Summary of COVID-19 Cases According to Wilayats}

To investigate which Wilayat of the three was the most affected by the Covid-19 outbreak and spread, we performed a statistical analysis of the three Wilayats. In Al Buraimi, $564(60.7 \%)$ of the patients were between 30-59 years, while Al Sunaina and Mahdha had $6(54.6 \%)$, 21(58.3\%), respectively of their patients in the same age group, and this formed the significant age group being infected at $591(60.5 \%)$. Similarly, the male at $\mathrm{Al}$ Buraimi were 693 (74.5\%), Al Sunaina 11 (100\%), and Mahdha at $34(94.4 \%)$. The total male sample was 738 (75.5\%). The hospital admission was as follows; Al Buraimi at $110(11.8 \%)$, Al Sunaina at $0 \%$, and Mahdha at 4(11.1\%). The ICU admission at Al Buraimi was 25 (2.7\%), Al Sunaina $0 \%$ and Mahdha $0 \%$. The cases whose outcome was death were distributed as follows; Al Buraimi 14 (1.5\%), Al Sunaina 0\%, and Mahdha 1 (2.8\%). Other demographical and epidemiological information based on the three Wilayat are shown in the Table 10.

Table 10. Summary Table of COVID-19 Cases According to Wilayat, Demographic Characteristics, Risk Factor, Admission, ICU Admission and Outcome, Al-Buraimi, Oman, February to August 2020

\begin{tabular}{|c|c|c|c|c|c|c|c|c|c|}
\hline \multirow{2}{*}{ Variables } & & \multicolumn{2}{|c|}{ Al Buraimi } & \multicolumn{2}{|c|}{ Al Sunaina } & \multicolumn{2}{|c|}{ Mahdha } & \multicolumn{2}{|c|}{ Total } \\
\hline & & $\mathbf{N}$ & $\%$ & $\mathbf{N}$ & $\%$ & $\mathbf{N}$ & $\%$ & $\mathbf{N}$ & $\%$ \\
\hline \multirow{6}{*}{ Age Category } & 0-4 Years & 13 & $1.4 \%$ & 0 & $0.0 \%$ & 0 & $0.0 \%$ & 13 & $1.3 \%$ \\
\hline & 5-14 Years & 26 & $2.8 \%$ & 0 & $0.0 \%$ & 0 & $0.0 \%$ & 26 & $2.7 \%$ \\
\hline & 15-29 Years & 268 & $28.8 \%$ & 5 & $45.5 \%$ & 14 & $38.9 \%$ & 287 & $29.4 \%$ \\
\hline & 30-59 Years & 564 & $60.7 \%$ & 6 & $54.6 \%$ & 21 & $58.3 \%$ & 591 & $60.5 \%$ \\
\hline & 60 Years and Above & 59 & $6.3 \%$ & 0 & $0.0 \%$ & 1 & $2.8 \%$ & 60 & $6.1 \%$ \\
\hline & Mean & 35.8 & & 31.5 & & 32.8 & & 35.6 & \\
\hline \multirow{2}{*}{$\operatorname{Sex}$} & Male & 693 & $74.5 \%$ & 11 & $100.0 \%$ & 34 & $94.4 \%$ & 738 & $75.5 \%$ \\
\hline & Female & 237 & $25.5 \%$ & 0 & $0.0 \%$ & 2 & $5.6 \%$ & 239 & $24.5 \%$ \\
\hline \multirow{2}{*}{ Nationality } & Omani & 534 & $57.4 \%$ & 1 & $9.1 \%$ & 15 & $41.7 \%$ & 550 & $56.3 \%$ \\
\hline & Non-Omani & 396 & $42.6 \%$ & 10 & $90.9 \%$ & 21 & $58.3 \%$ & 427 & $43.7 \%$ \\
\hline \multirow{2}{*}{ Risk Factor } & Yes & 227 & $24.4 \%$ & 0 & $0.0 \%$ & 5 & $13.9 \%$ & 232 & $23.8 \%$ \\
\hline & No & 703 & $75.6 \%$ & 11 & $100.0 \%$ & 31 & $86.1 \%$ & 745 & $76.3 \%$ \\
\hline \multirow{2}{*}{$\begin{array}{l}\text { Hospital } \\
\text { Admission }\end{array}$} & Yes & 110 & $11.8 \%$ & 0 & $0.0 \%$ & 4 & $11.1 \%$ & 114 & $11.7 \%$ \\
\hline & No & 0 & $88.2 \%$ & 11 & $100.0 \%$ & 32 & $88.9 \%$ & 863 & $88.3 \%$ \\
\hline \multirow{3}{*}{$\begin{array}{l}\text { ICU } \\
\text { Admission }\end{array}$} & Yes & 25 & $2.7 \%$ & 0 & $0.0 \%$ & 0 & $0.0 \%$ & 25 & $2.6 \%$ \\
\hline & No & 905 & $97.3 \%$ & 11 & $100.0 \%$ & 36 & $100.0 \%$ & 952 & $97.4 \%$ \\
\hline & Recovered & 916 & $98.5 \%$ & 11 & $100.0 \%$ & 35 & $97.2 \%$ & 962 & $98.5 \%$ \\
\hline \multirow[t]{2}{*}{ Outcome } & Died & 14 & $1.5 \%$ & 0 & $0.0 \%$ & 1 & $2.8 \%$ & 15 & $1.5 \%$ \\
\hline & TOTAL & 930 & $100.0 \%$ & 11 & $100.0 \%$ & 36 & $100.0 \%$ & 977 & $100.0 \%$ \\
\hline
\end{tabular}




\section{Discussion}

This analytical cross-sectional study provides a summary of epidemiological and clinical features of people with SARS-CoV-2 infection and the various risk factors associated with the need for hospitalization and critical outcome in the three wilayats of Al Buraimi Governorate. The data also shows the burden caused by the outbreak on both the healthcare facilities and the individuals involved.

As the study shows, there was a significant difference based on the sexes of the patients diagnosed with covid-19 from February to August 2020, where 738 (75.5\%) patients were male, and 239(24.5\%) were female. Even though we could not definitively determine why the rate of reported cases was significantly high in men as compared to women, we would wish to acknowledge a preceding study by Takahashi et al., 2020 which noted that the male patients have higher plasma levels of innate immune cytokines, while female patients exhibited more aggressive $\mathrm{T}$ cell activation than male patients. Such findings could be advanced further to show with certainty why there are clear differences in the severity of covid-19 infections (Takahashi et al., 2020). Another possible explanation is the higher prevalence of high-risk behavior, including smoking, higher prevalence of underlying comorbidities, and others that are yet to be fully defined by biologic differences (Palaiodimos et al., 2020).

There are several possible reasons why this study's results show lower disease severity in the three Wilayats studied. First, the initial containment effort in Oman included the admission and home confinement of all persons who tested positive even in the absence of symptoms. This was done at a time when most of the patients were asymptomatic (Khamis et al., 2020). Also, the mean age of our sample size shows that most of the initial infections were prevalent in the younger population, with the mean age being 35.7 years and other regions like New York recording a mean age of 62 years and Italy having a mean age of 47 years (Cummings et al., 2020; Palmieri et al., 2020). These countries had seen a high morbidity and mortality rate. Thus, due to this fact, we found that the prevalence of underlying chronic conditions to be relatively lower in our study than reported in other countries such as Brazil at a prevalence rate of $66.5 \%$ of heart diseases and $54.5 \%$ diabetes in covid- 19 patients (de Souza et al., 2020).

We were able to determine a pattern of risk factors that align with what the world health organization has classified as SARS-CoV-2 risk factors. The most severe risk factors included hypertension at a frequency of $106(10.8 \%)$, diabetes at $119(12.2 \%)$, age of more than 60 years at $53(5.4 \%)$, and heart disease at $36(3.7 \%)$. Other low-risk factors included Asthma at $27(2.8 \%)$, BMI $>40$ at $11(1.1 \%)$ patients, chronic kidney diseases at $10(1 \%)$, pregnancy at $17(1.7 \%)$ and Hypothyroidism at $10(1 \%)$. Other illnesses include chronic hematological disorder, chronic liver disease, chronic lung disease, immunocompromised, thyrotoxicosis, dyslipidemia, hepatitis $\mathrm{C}$, cancer, neurological disease, and rheumatologically diseases were found to have a frequency of 42 (4.3\%). Our studies' findings mirror other results from various parts of the world on the risk factors of Covid-19. For example, a study by Palaiodimos et al., 2020 summarized the risk factors of covid-19 as being old ( $\geq 65$ years old), male sex, having a higher BMI value $(>35 \mathrm{Kg} / \mathrm{m} 2)$, having comorbidities (e.g., hypertension, diabetes, cardiovascular and cerebrovascular diseases, etc.), and developing complications were vital risk factors for patients to develop severe conditions (Palaiodimos et al., 2020).

The risk of hospital admission and needing the intensive care facilities for people with the identified risk factors was very high. This is because from our sample size, we found that from 165 patients with risk factors, about 67 of them required hospital admission, and 14 proceeded into ICU admission. More so, the probability of death as an outcome for these patients was relatively high, was ten patients from the sample size of 232 subjects died, as compared to 5 from the sample size of 745 patients without underlying medical conditions. Besides, the data analysis showed that the highest likelihood of hospital admission in the category of high-risk patients was in patients having hypertension at a chi-square value of 96.33 , heart disease at 69.85 , diabetes at 63.31 , age more than 60 years 54.73, and chronic kidney disease at 22.90. However, conditions like pregnancy and Hypothyroidism with a p-value of 0.99 and 0.41 , respectively, had the least chances of hospital admission. The same findings were mirrored in the ICU's progression, where most patients needing the ICU services were aged more than 60 years, had chronic kidney disease, heart disease, diabetes, or hypertension. The need for ICU services for patients with Asthma, BMI $>40$, pregnancy, Hypothyroidism, and other conditions being the least people in need of ICU services. We found the overall need for ICU services to be at $25(2.6 \%)$, which is relatively lower than other observations in other studies. For example, Cummings et al.'s 2020 report on New York indicated an incidence of ICU admission at $22 \%$ (Cummings et al., 2020).

Risk factors are important to take into consideration in observing the progression and severity of disease as they tell the most likely reason involved in progression or severity. for instance, the age group of 60 years or above was reported to be involved in the progression of COVID-19 as observed by the previously conducted studies and the 
current study. This study found the continuous association of age 60 years or above with Hospitalization, ICU admission, and outcome. Further on, it showed the congruence with the results of previous studies stating the higher risk of poor outcome, greater severity in patient with age of 60 years or above (Liu et al., 2020). In comorbidities, diabetes is one of the most critical comorbidities which is potentially associated with hospitalization according to the current study (p-value < 0.001) and severity of COVID-19 (Rod et al., 2020). Diabetes is associated with infectious diseases and weaken or altered immunity which might be the explanation for its being a risk factor in COVID patients (Erener, 2020). Another study has labeled diabetes as an independent risk factor for morbidity and mortality in COVID-19 (Guo et al., 2020).

Hypertension is also a critical condition concerning COVID cases, researches carried out recently reported different results, some in favor of COVID-19 and some were non-favor results. SARS-CoV-2 requires ACE2 (angiotensin-converting enzyme 2) and activation of the renin-angiotensin system which is also the key system in blood pressure elevation in Hypertension. This reason is provided up until recently as an understanding of Hypertension involvement in COVID-19 progression and hospitalization, ICU admission, and outcome. But the studies have not yet confirmed this pathophysiological mechanism (Savoia et al., 2021). Our study has shown congruence with the recently conducted meta-analysis of hypertension as a risk factor which concluded that hypertension is an independent factor for increased risk of critical COVID-19 mortality, and it also reports an increase in in-hospital mortality (Du et al., 2021).

Heart disease is also a crucial underlying condition in COVID-19 patients as there are positive results of its likelihood of increasing the hospitalization of COVID patients, ICU admission, and potential to affect the outcome as can be seen in the results. In our study more than half of the COVID-19 patients with heart disease were hospitalized. Cardiovascular diseases are labelled as independent risk factor for COVID-19 patients leading to hospital admission, and ICU admission. Studies have found high mortality rate in patients with CVD, so the early vigilance and intervention should be taken in such patients ( $\mathrm{Li}$ et al., 2020). Asthma, being itself the respiratory problem, is thought of as a risk factor for the severity of COVID-19. The impact of Asthma on the progression and severity of COVID-19 and ICU admission and death are controversial with different results of different studies. Our study finds that Asthma is associated with Hospital admission but not with ICU admission and death. These results are the same as the study results reported by Calmes D. et al in their study (Calmes et al., 2021).

Initially, chronic kidney disease was not considered as the risk factor for severity of COVOID-19, but it is now considered as a potentially involved risk factor of COVID-19 mortality as observed and reported by the previous studies (Council \& Group, 2021). This current study also found the potential association of CKD with hospitalization, ICU admission, and death which shows consistency with previously reported results. CKD patients are observed to be at high risk with decreasing eGFR, hence, the lower the eGFR, the higher the risk of COVID-19 severity will be (Council \& Group, 2021). BMI is another parameter to be associated with the severity and progression of COVID-19. Obesity has been linked with respiratory problems due to its ability to develop acute respiratory distress syndrome (ARDS) which is the clinical manifestation of COVID-19 (Miyazawa, 2021). Our results show congruence with previous research as the association of BMI $>40$ with hospital admission and outcome type has been found statistically significant.

This study's primary strength was that it performed a data analysis on positive cases identified in Al Buraimi from February to August. This allowed for a comprehensive analysis of the COVID-19 burden and risk factors with minimal bias. The study's limitations were based on the reliance on existing records for data, where some important parameters needed to track the disease progression were absent. Also, most of the sample size was from Al Buraimi Governorate, which might introduce biases when using the results to generalize other regions of Oman. The lack of even spread of cases limits the results from being applied in other areas, and thus more research in other regions might be needed. Also, only a small proportion of the patients were over 60 years $60(6.1 \%)$ patients, and the small number insufficient depicting how severe age is a Covid-19 risk factor. Lastly, since a significant proportion of the population is asymptomatic, the chances are that the highest majority of the patient who sought treatment at medical facilities had symptoms. This might be biased when considering the burden and associated factors of the population COVID-19 in asymptomatic and symptomatic patients.

\section{Conclusion}

This study reinforces what the WHO organization classifies as risk factors of Covid-19. As the results show, the covid-19 cases were highly prevalent in Al Buraimi, as compared to Mahdha and Al Sunaina. This could be attributed to Al Buraimi being a meeting point of people from various regions in Oman and beyond. More so, in the effort to establish the risk factors of COVID-19, this study shows that hypertension, diabetes, age of more than 60 years, and heart disease pose a significant threat to people diagnosed with Covid-19. Besides, there was a 
correlation between the risk factors highlighted and hospitalization, ICU admission, and the outcome being death. This means that people with the indicated health conditions should be treated as a vulnerable population and protected with all means necessary from Covid infections. This is because these chronic illnesses put a significant burden on the patients and healthcare facilities as such patients have shown to frequently require hospital admission and ICU's. Thus, this study managed to accomplish its objectives as these findings are important in determining underlying conditions that can increased the likelihood of COVID-19 progression to critical levels.

\section{Recommendations}

This study makes the following recommendations:

1) The government and healthcare stakeholders should use the reports from this study to protect the people in the risk factors group and plan and equip the healthcare facilities to meet the needs of the vulnerable groups.

2) A large-scale study should be conducted on dealing with risk factors and developing the interventions to deal with them with the aim of reduction in the mortality and morbidity of COVID-19.

\section{Competing Interests Statement}

The authors declare that there are no competing or potential conflicts of interest.

\section{References}

Ahrenfeldt, L. J., Nielsen, C. R., Möller, S., Christensen, K., \& Lindahl-Jacobsen, R. (2020). Burden and prevalence of risk factors for severe COVID-19 disease in the ageing European population - A SHARE-based analysis. Res $S q$. https://doi.org/10.21203/rs.3.rs-73657/v1

Alandijany, T. A., Faizo, A. A., \& Azhar, E. I. (2020). Coronavirus disease of 2019 (COVID-19) in the Gulf Cooperation Council (GCC) countries: Current status and management practices. Journal of Infection and Public Health, 13(6), 839-842. https://doi.org/10.1016/j.jiph.2020.05.020

Azarpazhooh, M. R., Morovatdar, N., Avan, A., Phan, T. G., Divani, A. A., Yassi, N., . . Di Napoli, M. (2020). COVID-19 Pandemic and Burden of Non-Communicable Diseases: An Ecological Study on Data of 185 Countries. Journal of Stroke and Cerebrovascular Diseases, 29(9), 105089. https://doi.org/10.1016/j.jstrokecerebrovasdis.2020.105089

Boukhatem, N. (2020). COVID-19 update : what's going on? Journal of Materials and Environmental Science, 11.

Calmes, D., Graff, S., Maes, N., Frix, A. N., Thys, M., Bonhomme, O., .. S Schleich, F. (2021). Asthma and COPD Are Not Risk Factors for ICU Stay and Death in Case of SARS-CoV2 Infection. J Allergy Clin Immunol Pract, 9(1), 160-169. https://doi.org/10.1016/j.jaip.2020.09.044

Clark, A., Jit, M., Warren-Gash, C., Guthrie, B., Wang, H. H. X., Mercer, S. W., . . Eggo, R. M. (2020). Global, regional, and national estimates of the population at increased risk of severe COVID-19 due to underlying health conditions in 2020: a modelling study. Lancet Glob Health, 8(8), e1003-e1017. https://doi.org/10.1016/S2214-109X(20)30264-3

Council, E.-E., \& Group, E. W. (2021). Chronic kidney disease is a key risk factor for severe COVID-19: a call to action by the ERA-EDTA. Nephrology Dialysis Transplantation, 36(1), 87-94. https://doi.org/10.1093/ndt/gfaa314

Cummings, M. J., Baldwin, M. R., Abrams, D., Jacobson, S. D., Meyer, B. J., Balough, E. M., , . O'Donnell, M. R. (2020). Epidemiology, clinical course, and outcomes of critically ill adults with COVID-19 in New York City: a prospective cohort study. Lancet, 395(10239), 1763-1770. https://doi.org/10.1016/S0140-6736(20)31189-2

de Souza, W. M., Buss, L. F., Candido, D. d. S., Carrera, J.-P., Li, S., Zarebski, A. E., . . Faria, N. R. (2020). Epidemiological and clinical characteristics of the COVID-19 epidemic in Brazil. Nature Human Behaviour, 4(8), 856-865. https://doi.org/10.1038/s41562-020-0928-4

Du, Y., Zhou, N., Zha, W., \& Lv, Y. (2021). Hypertension is a clinically important risk factor for critical illness and mortality in COVID-19: A meta-analysis. Nutrition, Metabolism and Cardiovascular Diseases, 31(3), 745-755. https://doi.org/10.1016/j.numecd.2020.12.009

Erener, S. (2020). Diabetes, infection risk and COVID-19. Molecular Metabolism, 39, 101044. https://doi.org/10.1016/j.molmet.2020.101044

Guo, W., Li, M., Dong, Y., Zhou, H., Zhang, Z., Tian, C., . . Hu, D. (2020). Diabetes is a risk factor for the progression and prognosis of COVID-19. Diabetes/Metabolism Research and Reviews, 36(7), e3319. https://doi.org/10.1002/dmrr.3319 
Jo, M. W., Go, D. S., Kim, R., Lee, S. W., Ock, M., Kim, Y. E., . . Park, H. (2020). The Burden of Disease due to COVID-19 in Korea Using Disability-Adjusted Life Years. J Korean Med Sci, 35(21), e199. https://doi.org/10.3346/jkms.2020.35.e199

Khamis, F., Al Rashidi, B., Al-Zakwani, I., Al Wahaibi, A. H., \& Al Awaidy, S. T. (2020). Epidemiology of COVID-19 Infection in Oman: Analysis of the First 1304 Cases. Oman medical journal, 35(3), e145. https://doi.org/10.5001/omj.2020.60

Kraef, C., Juma, P., Kallestrup, P., Mucumbitsi, J., Ramaiya, K., \& Yonga, G. (2020). The COVID-19 Pandemic and Non-communicable Diseases-A Wake-up Call for Primary Health Care System Strengthening in Sub-Saharan Africa. $J$ Prim Care Community Health, 11, 2150132720946948. https://doi.org/10.1177/2150132720946948

Li, J., Guo, T., Dong, D., Zhang, X., Chen, X., Feng, Y., ... Wan, J. (2020). Defining heart disease risk for death in COVID-19 infection. QJM: An International Journal of Medicine, 113(12), 876-882. https://doi.org/10.1093/qjmed/hcaa246

Liu, Y., Mao, B., Liang, S., Yang, J.-W., Lu, H.-W., Chai, Y.-H., . . Shanghai Clinical Treatment Experts Group for, C. (2020). Association between age and clinical characteristics and outcomes of COVID-19. The European respiratory journal, 55(5), 2001112. https://doi.org/10.1183/13993003.01112-2020

Miyazawa, D. (2021). Why obesity, hypertension, diabetes, and ethnicities are common risk factors for COVID-19 and H1N1 influenza infections. Journal of Medical Virology, 93(1), 127-128. https://doi.org/10.1002/jmv.26220

Ogeng'o, J., Karau, P. B., Misiani, M., Cheruiyot, I., Olabu, B., \& Kariuki, B. N. (2020). Coronavirus Disease 2019 (COVID-19) set to increase burden of atherosclerotic cardiovascular disease in Kenya. Pan African Medical Journal, 35(2), 120. https://doi.org/10.11604/pamj.supp.2020.35.2.24762

Palaiodimos, L., Kokkinidis, D. G., Li, W., Karamanis, D., Ognibene, J., Arora, S., . . Mantzoros, C. S. (2020). Severe obesity, increasing age and male sex are independently associated with worse in-hospital outcomes, and higher in-hospital mortality, in a cohort of patients with COVID-19 in the Bronx, New York. Metabolism, 108, 154262. https://doi.org/10.1016/j.metabol.2020.154262

Palmieri, L., Andrianou, X., Bella, A., \& Bellino, S. (2020). EpiCentro- Portale di epidemiologia per fli operatori santiari. Retrieved from https://www.epicentro.iss.it/coronavirus/bollettino/Report-COVID-2019_20_ marzo_eng.pdf

Park, M. D. (2020). Sex differences in immune responses in COVID-19. Nature Reviews Immunology, 20(8), 461-461. https://doi.org/10.1038/s41577-020-0378-2

Rod, J. E., Oviedo-Trespalacios, O., \& Cortes-Ramirez, J. (2020). A brief-review of the risk factors for covid-19 severity. Rev Saude Publica, 54, 60. https://doi.org/10.11606/s1518-8787.2020054002481

Savoia, C., Volpe, M., \& Kreutz, R. (2021). Hypertension, a Moving Target in COVID-19. Circulation Research, 128(7), 1062-1079. https://doi.org/10.1161/CIRCRESAHA.121.318054

Takahashi, T., Ellingson, M. K., Wong, P., Israelow, B., Lucas, C., Klein, J., . . Y Yale, I. R. T. (2020). Sex differences in immune responses that underlie COVID-19 disease outcomes. Nature, 588(7837), 315-320. https://doi.org/10.1038/s41586-020-2700-3

World Health Organization [WHO]. (2017). Oman Country Profile. Retrieved August 23rd from https://www.who.int/beat-ncds/countries/oman/en/

World Health Organization [WHO]. (2020). Attacks on Healthcare in the Context of COVID-19. Retrieved July 30th from https://www.who.int/news-room/feature-stories/detail/attacks-on-health-care-in-the-contextof-covid-19

World Meters. (2020). Oman Coronavirus: 83,769 cases and 609 deaths. Retrieved August 20th from https://www.worldometers.info/coronavirus/country/oman/

\section{Copyrights}

Copyright for this article is retained by the author(s), with first publication rights granted to the journal.

This is an open-access article distributed under the terms and conditions of the Creative Commons Attribution license (http://creativecommons.org/licenses/by/4.0/). 